\title{
Colloque international
}

\section{De l’interdisciplinarité à la transdisciplinarité ?}

Nouveaux enjeux, nouveaux objets de la recherche en littérature et sciences humaines

\section{Jeudi 27, vendredi 28 et samedi 29 novembre 2014}

\section{Université Paris-Est Créteil}

(salle des thèses ; métro ligne 8 , station Créteil-Université)

Responsables scientifiques : Laure de Nervaux-Gavoty, Sylvie Le Moël, Guillaume Marche

Jeudi 27 novembre: Disciplinarité, interdisciplinarité, transdisciplinarité : nouveaux enjeux, nouveaux objets

9h00: Ouverture du colloque par Jeanne-Marie Boivin, doyen de la Faculté des Lettres, Langues et Sciences Humaines, et Graciela Villanueva, directrice d'IMAGER.

\section{$9 \mathrm{~h} 30$ : Conférence plénière}

Frédéric Darbellay, Professeur associé en Inter- et Transdisciplinarité à l'Institut Universitaire Kurt Bösch (IUKB, Sion, Suisse), auteur notamment de La circulation des savoirs. Interdisciplinarité, concepts nomades, analogies, métaphores (Peter Lang, 2012) et de L'interdisciplinarité racontée. Chercher hors frontières, vivre l'interculturalité (Peter Lang, 2014 - avec Violaine Lemay)

$11 \mathrm{~h} 00$ : Atelier $\mathrm{n}^{\circ} 1$ - Frontières et territoires disciplinaires

Présidence : Jean-Paul Rocchi (Université Paris-Est Marne-la-Vallée)

Isabelle Alfandary (Université Sorbonne Nouvelle Paris 3) «La littérature est-elle une discipline ? "

Lucile Dumont (Ecole des Hautes Etudes en Sciences Sociales) «L'aventure théorique des études littéraires au prisme de l'interdisciplinarité »

Anne-Claire Collier (Université Paris Ouest Nanterre) «Territoires et frontières: le débat postcolonial au prisme des disciplines »

$14 \mathrm{~h} 00$ : Atelier $\mathrm{n}^{\circ} 2$ - Enjeux éthiques et épistémologiques de la transdisciplinarité

Présidence : Bernard Banoun (Université Paris-Sorbonne)

Hans-Jürgen Lüsebrink (Université de Sarrebruck) «Entre sémantique et analyse du discours- discours, concepts et 'idées' comme objets d'une approche interdisciplinaire en lettres et sciences humaines "

Sarah Alharbi (Université de Montréal) « Le dialogue entre les disciplines comme paradigme du savoir en littérature et sciences humaines: sur l'aspect didactique des travaux interdisciplinaires du groupe de recherche Poetik und Hermeneutik à l'école de Constance (1963-1994) »

Anne Chalard-Fillaudeau (Université Paris 8 Vincennes Saint-Denis) « La transdisciplinarité et le principe de responsabilité » 
$16 \mathrm{~h} 00$ : Atelier $\mathrm{n}^{\circ} 3$ - Constitution de nouveaux objets d'étude

Présidence : Jagna Oltarzewska (Université Paris-Sorbonne)

David Ledent (Université de Liège) «La sociologie par la littérature: une approche transdisciplinaire »

Anne Hurault-Paupe (Université Paris 13) «Buffy Studies et Porn Studies : histoire et enjeux de deux champs d'étude émergents"

Anne Lagny (ENS de Lyon) « La transdisciplinarité dans le champ des études piétistes »

$17 \mathrm{~h} 30$ : cocktail

$20 \mathrm{h00}$ : spectacle au Conservatoire Marcel-Dadi (Créteil)

Vendredi 28 novembre : La transdisciplinarité mise en pratique

$9 \mathrm{~h} 30$ : Conférence plénière

Basarab Nicolescu, physicien et philosophe, Professeur à la Faculté d'études européennes de I'Université Babes-Bolyai (Cluj-Napoca, Roumanie), Président du Centre International de Recherches et études Transdisciplinaires (CIRET), auteur notamment de Qu'est-ce que la réalité ? (Liber, 2009) et de Nous, la particule et le monde (Le Rocher, 2002)

$11 \mathrm{~h} 00$ : Atelier $n^{\circ} 4$ - Méthodologies transdisciplinaires

Présidence : Vincent Ferré (Université Paris-Est Créteil)

Isabelle Matamoros (Université Lumière Lyon 2 et Université Paris Descartes) " La lecture des femmes, entre histoire, littérature et études de genre: à objet interdisciplinaire, méthode transdisciplinaire ? "

Laura Péaud (Université Lumière Lyon 2) «L'enjeu disciplinaire pour la géographie : mise en perspective historique "

$14 \mathrm{~h} 00$ : Atelier $\mathrm{n}^{\circ} 5$ - Apports et limites de la transdisciplinarité (1)

Présidence : Graciela Villanueva (Université Paris-Est Créteil)

Alfredo Vega (Université Paris 1 Panthéon-Sorbonne) « Une discipline en quête d'identité. Défis transdisciplinaires pour la conservation-restauration de biens culturels »

Angélica Rigaudière (Université de Reims Champagne-Ardenne) "Promesses de la communication et ouverture disciplinaire: programmes de revues numériques sur la musique »

$15 \mathrm{~h} 30$ : Atelier $\mathrm{n}^{\circ} 6$ - Apports et limites de la transdisciplinarité (2)

Présidence : Donna Kesselman (Université Paris-Est Créteil)

Muriel Montagut (Université de Perpignan Via Domitia) «La transdisciplinarité du fait totalitaire »

Marc Riedel (Université Paris Dauphine) et Eric Brousse (Service Départemental d'Incendie et de Secours de Saône et Loire) "Rythmes de travail, pénibilité et horaires atypiques: une recherche transdisciplinaire en situation réelle de travail chez les sapeurs-pompiers » 
17 h00 : Table-ronde - Enjeux institutionnels et didactiques de la transdisciplinarité Présidence : Thierry Gallèpe (Université Bordeaux Montaigne)

Brigitte Marin, Directrice de L'École Supérieure du Professorat et de l'Éducation (ESPE) de l'académie de Créteil

Olivier Mérot, Directeur du Conservatoire à Rayonnement Départemental Marcel-Dadi de Créteil

Yves Laberge, sociologue, Directeur des collections "L'espace public » et "Cinéma et société » aux Presses de l'Université Laval (Canada) (sous réserve)

Jean-Marc Quaranta, Maître de conférences en littérature française et création littéraire à I'Université d'Aix-Marseille, responsable du D.U. "Formateur en ateliers d'écriture » et de la licence de lettres modernes "Création littéraire et cinématographique »

\section{Samedi 29 novembre : L'art au prisme de la transdisciplinarité}

\section{$9 \mathrm{~h} 30$ : Conférence plénière}

Thierry Paquot, philosophe, Professeur des universités à l'Institut d'urbanisme de Paris (Université Paris-Est Créteil), éditeur de la revue Urbanisme, producteur de "Côté ville" dans l'émission Métropolitains de France-Culture, responsable scientifique du programme "La forme d'une ville » au Forum des Images (Paris), auteur notamment de : Éloge du luxe. De l'utilité de l'inutile (Bourin-éditeur, 2005), Des corps urbains. Sensibilités entre béton et bitume, (Autrement, 2006), Terre urbaine. Cinq défis pour le devenir urbain de la planète (La Découverte, 2006) et Petit manifeste pour une écologie existentielle (Bourin-éditeur, 2007)

11 h00 : Atelier $n^{\circ} 7$ - Penser l'art aujourd'hui

Présidence : Caroline Zekri (Université Paris-Est Créteil)

Marie-Thérèse Mourey (Université Paris-Sorbonne) « La danse, un nouvel objet de recherche transdisciplinaire ? »

Danielle Boutet (Université du Québec à Rimouski) "L'art aujourd'hui et la transdisciplinarité: mise en oeuvre de savoirs artistiques dans des espaces transdisciplinaires »

Viviane Huys (Université Bordeaux Montaigne) «La notion de contrainte épistémologique : les raisons de l'indiscipline $"$

$14 \mathrm{~h} 00$ : Atelier $\mathrm{n}^{\circ} 8$-transdisciplinarité et transartialité

Présidence : Marielle Silhouette (Université Paris Ouest Nanterre)

Hilda Inderwildi (Université de Toulouse Jean Jaurès) "L'objet théâtral au croisement des arts et des disciplines. État des lieux »

Ivan Jiménez (Université Paris-Est Créteil) « Danse et transdisciplinarité. Inxilio et la violence en Colombie »

$15 \mathrm{~h} 00$ : Pascal Poyet (poète) et Françoise Goria (artiste) : lecture-écrans-performés et accrochage des éditions contrat maint 